\title{
Two Types of Isotropic Vector Play Models and Their Rotational Hysteresis Losses
}

$\operatorname{AUTHOR}(\mathrm{S}):$

Matsuo, Tetsuji; Shimasaki, Masaaki

\section{CITATION:}

Matsuo, Tetsuji ... [et al]. Two Types of Isotropic Vector Play Models and Their Rotational Hysteresis Losses. IEEE TRANSACTIONS ON MAGNETICS 2008, 44(6): 898-901

\section{ISSUE DATE:}

2008-06

URL:

http://hdl.handle.net/2433/84542

\section{RIGHT:}

(c) 2008 IEEE. Personal use of this material is permitted. However, permission to reprint/republish this material for advertising or promotional purposes or for creating new collective works for resale or redistribution to servers or lists, or to reuse any copyrighted component of this work in other works must be obtained from the IEEE. 


\title{
Two Types of Isotropic Vector Play Models and Their Rotational Hysteresis Losses
}

\author{
Tetsuji Matsuo $^{1}$ and Masaaki Shimasaki ${ }^{2}$ \\ ${ }^{1}$ Department of Electrical Engineering, Graduate School of Engineering, Kyoto University, Kyoto, 615-8510 Japan \\ ${ }^{2}$ Fukui University of Technology, Fukui, Japan
}

This paper presents a comparison of two types of isotropic vector play models and their generalized models. The first vector model is represented by a superposition of scalar play models. The other type is given by a geometrical vectorization of play hysteron. The rotational hysteresis losses of both models are discussed. A method is proposed to adjust the simulated rotational hysteresis loss to a measured loss.

Index Terms-Isotropic vector hysteresis, rotational hysteresis loss, superposition, vector play hysteron.

\section{INTRODUCTION}

$\mathbf{T}$ THE play model [1], [2] is an efficient hysteresis model that can be implemented more simply than the Preisach model [3]. Yet, the play model with input-independent shape functions has been proven equivalent to the classical Preisach model [4]. A previous study [5] has shown that the play model with inputdependent shape functions is mathematically equivalent to the nonlinear Preisach model proposed by Mayergoyz [3].

Two vector versions of the play model have been proposed in previous studies: one is a geometric extension of the scalar model [1], [6], [7], whereas the other is a superposition of scalar models along the azimuthal direction [8], [9].

This paper discusses rotational hysteresis losses of the two types of two-dimensional (2D) isotropic vector play models. Both models are generalized using weighting functions to control their rotational hysteresis losses. A method to determine the weighting function is proposed to adjust the simulated rotational hysteresis loss to a measured loss.

\section{SCAlar Play Model AND ITS Hysteresis LosS}

The play model usually gives a hysteretic output of magnetic flux density $B$ from an input of magnetic field $H$. However, it is also able to provide an output $H$ from an input $B$ similarly to the Preisach model using the inverse distribution function method [10]. The play model describes a hysteretic function having input $B$ as

$$
H=P(B)=\int_{0}^{B_{\mathrm{S}}} f\left(\zeta, p_{\zeta}(B)\right) \mathrm{d} \zeta
$$

where $p_{\zeta}$ is a play hysteron operator having width $\zeta, f$ is an input-independent shape function, and $B_{\mathrm{S}}$ is the saturation magnetic flux density. The play hysteron operator is given as

$$
p_{\zeta}(B)=\max \left(\min \left(p_{\zeta}^{0}, B+\zeta\right), B-\zeta\right)
$$

where $p_{\zeta}^{0}$ is the value of $p_{\zeta}$ at the previous time-point. Fig. 1 illustrates characteristics of the play hysteron.

The parameter $B_{\mathrm{S}}$ is chosen as the magnetic flux density at which the saturation of magnetization becomes significant. It is

Digital Object Identifier 10.1109/TMAG.2007.914852

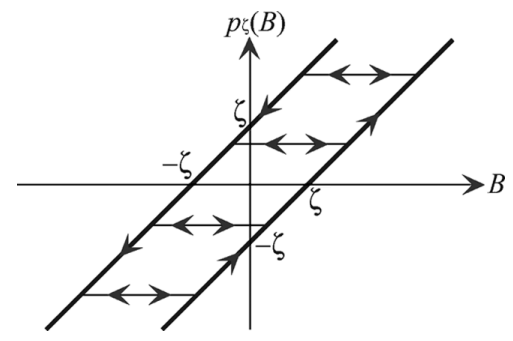

Fig. 1. Play hysteron operator.

assumed that $P(B)$ does not exhibit hysteretic properties for $|B| \geq B_{\mathrm{S}}$. To ensure that $P(B)$ is a single-valued function for $|\bar{B}| \geq B_{\mathrm{S}}, f(\zeta, p)$ is set to be constant $(\partial f / \partial p=0)$ for $|p| \geq B_{\mathrm{S}}-\zeta$.

For an alternating input with amplitude $B_{\mathrm{a}}$, the play model $P(B)$ yields a hysteresis loss $L_{\mathrm{alt}}\left(B_{\mathrm{a}}\right)$ per cycle of

$$
L_{\text {alt }}\left(B_{\mathrm{a}}\right)=\oint_{B_{\mathrm{a}}} P(B) \mathrm{d} B=-4 \int_{0}^{B_{\mathrm{m}}} f\left(\zeta, B_{\mathrm{a}}-\zeta\right) \zeta \mathrm{d} \zeta
$$

where $f(\zeta,-B)=-f(\zeta, B)$ is assumed and

$$
B_{\mathrm{m}}=\min \left(B_{\mathrm{a}}, B_{\mathrm{S}}\right) .
$$

The minus sign in (3) is required because the input is not $H$ but $B$.

\section{SuPERPosition of SCALAR Play Models}

\section{A. Superposition of Scalar Play Models}

Similarly to the vector Preisach model proposed by Mayergoyz [3], a 2D isotropic vector play model can be constructed using the superposition of scalar play models [8], [9]

$$
\boldsymbol{H}=\boldsymbol{P}_{\mathrm{S}}(\boldsymbol{B})=\int_{-\pi / 2}^{\pi / 2} \boldsymbol{e}_{\varphi} P_{2}\left(|\boldsymbol{B}| \cos ^{\frac{1}{n}}(\theta-\varphi)\right) \mathrm{d} \varphi .
$$

Here, $P_{2}(B)$ is a scalar play model, $\boldsymbol{e}_{\varphi}$ is the unit vector along the $\varphi$-direction, $\theta$ is the angle of $\boldsymbol{B}$, and $n$ is a projection parameter for input $\boldsymbol{B}$ to the $\varphi$-direction; $\cos ^{1 / n} \phi$ denotes 
$|\cos \phi|^{1 / n} \operatorname{sign}(\cos \phi)$ for simplicity of expression. The identification method of the vector model (5) is given in [9] (see Appendix).

When a rotational input

$$
\boldsymbol{B}=\left(B_{\mathrm{a}} \cos \omega t, B_{\mathrm{a}} \sin \omega t\right)
$$

is given, the vector model (5) yields the hysteresis loss

$$
L_{\mathrm{rot}}\left(B_{\mathrm{a}}\right)=\pi \oint_{B_{\mathrm{a}}} P_{2}\left(B_{\mathrm{a}}\left(\frac{B}{B_{\mathrm{a}}}\right)^{\frac{1}{n}}\right) \mathrm{d} B .
$$

When $n=1, L_{\text {rot }}\left(B_{\mathrm{a}}\right)$ becomes

$$
L_{\mathrm{rot}}\left(B_{\mathrm{a}}\right)=\pi L_{2 \mathrm{alt}}\left(B_{\mathrm{a}}\right)
$$

where $L_{2 \text { alt }}$ is the alternating hysteresis loss given by $P_{2}$ as (3). Because $L_{2 \text { alt }}\left(B_{\mathrm{a}}\right)$ is constant for $B_{\mathrm{a}} \geq B_{\mathrm{S}}, L_{\mathrm{rot}}\left(B_{\mathrm{a}}\right)$ is also constant for $B_{\mathrm{a}} \geq B_{\mathrm{S}}$. This constant rotational hysteresis loss is not observed in actual ferromagnetic materials.

\section{B. Generalized Vector Model With Weighting Function}

Similarly to the scalar play model with input-dependent shape functions [11], the vector play model is generalized as

$$
\boldsymbol{P}_{\mathrm{Sw}}(\boldsymbol{B})=w(|\boldsymbol{B}|) \int_{-\pi / 2}^{\pi / 2} \boldsymbol{e}_{\varphi} P_{2}\left(|\boldsymbol{B}| \cos ^{\frac{1}{n}}(\theta-\varphi)\right) \mathrm{d} \varphi
$$

where $w(B)$ is a weighting function. Equations (5) and (9) imply that $P_{\mathrm{Sw}}(\boldsymbol{B}) / w(|\boldsymbol{B}|)=P_{\mathrm{S}}(\boldsymbol{B})$ is the vector play model (5). For the rotational input (6), $P_{\mathrm{Sw}}(\boldsymbol{B})$ yields a (rate-independent) hysteresis loss of

$$
L_{\mathrm{wrot}}\left(B_{\mathrm{a}}\right)=w\left(B_{\mathrm{a}}\right) L_{\text {rot }}\left(B_{\mathrm{a}}\right)
$$

where $L_{\text {rot }}$ is given by (7). When $n=1, L_{\text {wrot }}$ becomes

$$
L_{\text {wrot }}\left(B_{\mathrm{a}}\right)=\pi w\left(B_{\mathrm{a}}\right) L_{2 \text { alt }}\left(B_{\mathrm{a}}\right) .
$$

The rotational hysteresis loss depends strongly on $w(B)$. However, $L_{\mathrm{wrot}}\left(B_{\mathrm{a}}\right)$ is not proportional to $w\left(B_{\mathrm{a}}\right)$ for $B_{\mathrm{a}}<B_{\mathrm{S}}$ because the scalar model $P_{2}$ and its alternating hysteresis loss $L_{2 \text { alt }}$ also depend on the choice of $w(B)$.

When $|B| \geq B_{\mathrm{S}}, w(B)$ does not affect the scalar hysteretic property of $P_{2}$. Accordingly, $w(B)$ can be determined to control directly the rotational hysteresis loss $L_{\mathrm{wrot}}\left(B_{\mathrm{a}}\right)$ for $B_{\mathrm{a}} \geq$ $B_{\mathrm{S}}$. For example, when $n=1$, because $L_{\mathrm{rot}}\left(B_{\mathrm{a}}\right)$ is constant, $L_{\text {wrot }}\left(B_{\mathrm{a}}\right)$ is proportional to $w\left(B_{\mathrm{a}}\right)$ as

$$
L_{\mathrm{wrot}}\left(B_{\mathrm{a}}\right)=\frac{w\left(B_{\mathrm{a}}\right) L_{\mathrm{wrot}}\left(B_{\mathrm{S}}\right)}{w\left(B_{\mathrm{S}}\right)}\left(B_{\mathrm{a}} \geq B_{\mathrm{S}}\right) .
$$

\section{GeOMETRICAl VectorizATION OF the Play Hysteron}

\section{A. Vectorization of the Play Hysteron}

The play hysteron (2) is geometrically vectorized [6] as

$$
\boldsymbol{p}_{\zeta}(\boldsymbol{B})=\boldsymbol{B}-\frac{\boldsymbol{B}-\boldsymbol{p}_{\zeta}^{0}}{\max \left(\frac{\left|\boldsymbol{B}-\boldsymbol{p}_{\zeta}^{0}\right|}{\zeta}, 1\right)}
$$

where $\boldsymbol{p}_{\zeta}^{0}$ is the value of $\boldsymbol{p}_{\zeta}$ at the previous time-point. The geometrical meaning of the vector play hysteron is illustrated in
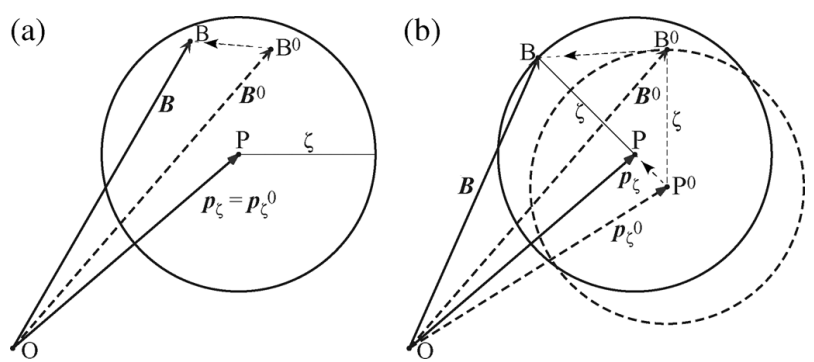

Fig. 2. Behavior of vector play hysteron: (a) $\left|\boldsymbol{B}-\boldsymbol{p}_{\zeta}^{0}\right| \leq \zeta$ and (b) $\left|\boldsymbol{B}-\boldsymbol{p}_{\zeta}^{0}\right|>\zeta$.

Fig. 2. A point $\mathrm{B}(\overrightarrow{\mathrm{OB}}=\boldsymbol{B})$ is located in a circle of which the center and radius are $\mathrm{P}\left(\overrightarrow{\mathrm{OP}}=\boldsymbol{p}_{\zeta}\right)$ and $\zeta$, respectively. When $\mathrm{B}$ moves inside the circle, $\mathrm{P}$ does not move [Fig. 2(a)]. When B reaches the circumference of the circle, the circle and its center $P$ move together with B [Fig. 2(b)].

Using this vector play hysteron, a vector play model [6], [7] is given as

$$
\begin{aligned}
\boldsymbol{P}_{\mathrm{G}}(\boldsymbol{B}) & =\int_{0}^{B_{\mathrm{S}}} \boldsymbol{f}\left(\zeta, \boldsymbol{p}_{\zeta}(\boldsymbol{B})\right) \mathrm{d} \zeta \\
\boldsymbol{f}(\zeta, \boldsymbol{p}) & =f(\zeta,|\boldsymbol{p}|) \frac{\boldsymbol{p}}{|\boldsymbol{p}|} .
\end{aligned}
$$

This vector model is simpler than the vector model (5) having an integration along the $\varphi$-direction that is computationally expensive.

For unidirectional inputs, the vector model (14) is reduced to the scalar model (1) having the same shape function $f(\zeta, p)$. This means that $f(\zeta, p)$ can be determined only from unidirectional measured data.

For rotational input with amplitude $B_{\mathrm{a}}$, the vector model (14) yields a (rate-independent) hysteresis loss [7] of

$$
L_{\mathrm{rot}}\left(B_{\mathrm{a}}\right)=-2 \pi \int_{0}^{B_{\mathrm{m}}} f\left(\zeta, \sqrt{B_{\mathrm{a}}^{2}-\zeta^{2}}\right) \zeta \mathrm{d} \zeta .
$$

\section{B. Generalized Vector Model With Weighting Function}

Serpico, d'Aquino et al. [7] have proposed an attractive generalization of the vector play model (14) and have discussed its properties including the rotational hysteresis loss. This paper proposes another simple useful generalization.

Similarly to the generalized model (9) using $w(B)$, the vector model (14) is generalized as

$$
\boldsymbol{P}_{\mathrm{Gw}}(\boldsymbol{B})=w(|\boldsymbol{B}|) \int_{0}^{B_{\mathrm{S}}} \boldsymbol{f}\left(\zeta, \boldsymbol{p}_{\zeta}(\boldsymbol{B})\right) \mathrm{d} \zeta .
$$

This model yields a rotational hysteresis loss of

$$
L_{\mathrm{wrot}}\left(B_{\mathrm{a}}\right)=-2 \pi w\left(B_{\mathrm{a}}\right) \int_{0}^{B_{\mathrm{m}}} f\left(\zeta, \sqrt{B_{\mathrm{a}}^{2}-\zeta^{2}}\right) \zeta \mathrm{d} \zeta .
$$

The rotational hysteresis loss is controlled by $w(B)$. For example, because $L_{\text {rot }}\left(B_{\mathrm{a}}\right)$ is constant for $B_{\mathrm{a}} \geq B_{\mathrm{S}}, L_{\mathrm{wrot}}(B)$ is proportional to $w(B)$ as in (12). 


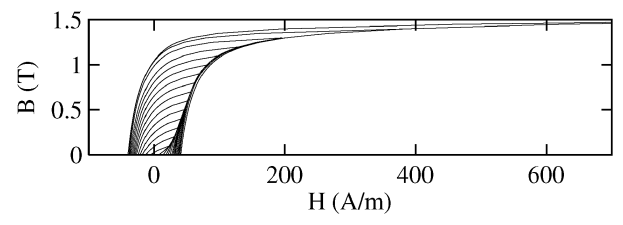

Fig. 3. Measured unidirectional property (averaged along the $\varphi$-direction).

\section{COMPARISON OF Vector MOdels}

\section{A. Identification From Unidirectional Property}

The hysteretic properties of a non-oriented silicon steel sheet, JIS: $35 \mathrm{~A} 300$ are measured using a rotational single sheet tester [12]. The anisotropic property is averaged along the azimuthal direction to obtain an isotropic property for numerical simulations. Fig. 3 shows an averaged unidirectional property that is used to identify scalar and vector play models.

The weighting function below is examined

$$
w(B)=1-c\left(\frac{B}{B_{\mathrm{S}}}\right)^{2}
$$

where $c$ is a constant. When $c=0$, the vector models $\boldsymbol{P}_{\mathrm{Sw}}$ of (9) and $\boldsymbol{P}_{\mathrm{Gw}}$ of (17) are reduced to $\boldsymbol{P}_{\mathrm{S}}$ of (5) and $\boldsymbol{P}_{\mathrm{G}}$ of (14), respectively.

First, the scalar play model (1) discretized with 64 hysterons is identified using the method described in [11]. Owing to the lack of measured data greater than $1.6 \mathrm{~T}, B_{\mathrm{S}}$ is set to $1.6 \mathrm{~T}$, despite this being small compared with the saturation magnetization of silicon steel sheet. The shape function of the scalar model is used directly for $\boldsymbol{P}_{\mathrm{Gw}}$. The vector model $\boldsymbol{P}_{\mathrm{Sw}}$ is identified from the scalar model as in the Appendix. The integration along the $\varphi$-direction is performed using the midpoint rule with 40 integration points. Because of this azimuthal integration, $\boldsymbol{P}_{\mathrm{Sw}}$ requires about 14 times more computation time than $\boldsymbol{P}_{\mathrm{Gw}}$.

Fig. 4(a) shows BH loops obtained by the vector play model $\boldsymbol{P}_{\mathrm{Gw}}$ with $c=0.5$ and of which the unidirectional property coincides with the property of the scalar play model. Fig. 4(b) and (c) shows BH loops given by the vector play model $\boldsymbol{P}_{\mathrm{Sw}}$ with $n=1,3$, respectively, and $c=0.5$. Fig. 4 shows that both vector models reconstruct $\mathrm{BH}$ loops exactly.

\section{B. Vector Property for Rotational Input}

Vector hysteretic properties of the vector models for rotational inputs are examined. Fig. 5(a) and (b) shows the rotational hysteresis losses yielded by $\boldsymbol{P}_{\mathrm{Gw}}$ and $\boldsymbol{P}_{\mathrm{Sw}}(n=1)$, respectively, Fig. 5(c) is given by $\boldsymbol{P}_{\mathrm{S}}\left(\boldsymbol{P}_{\mathrm{Sw}}\right.$ with $\left.c=0\right)$ and $n=$ $1,3,5$. For comparison, the measured rotational hysteresis loss $L_{\text {rot }}^{\text {measured }}$ is also shown.

Fig. 5(d) shows the phase lag of $\boldsymbol{B}$ to $\boldsymbol{H}$ with $c=0.5$, together with the measured average phase lag $\beta_{\text {lag }}$ given by (20)

$$
\beta_{\text {lag }}=\tan ^{-1} \frac{\left|\int_{0}^{2 \pi} \boldsymbol{H} \times \boldsymbol{B d}(\omega t)\right|}{\int_{0}^{2 \pi} \boldsymbol{H} \cdot \boldsymbol{B d}(\omega t)} .
$$

Fig. 5(a) and (b) shows that $\boldsymbol{P}_{\mathrm{G}}\left(\boldsymbol{P}_{\mathrm{Gw}}\right.$ with $\left.c=0\right)$ and $\boldsymbol{P}_{\mathrm{S}}$ with $n=1$ yield constant hysteresis losses for $B_{\mathrm{a}}>1.6 \mathrm{~T}$ because $B_{\mathrm{S}}$ is set to $1.6 \mathrm{~T}$. The vector model $\boldsymbol{P}_{\mathrm{S}}$ with $n=3,5$
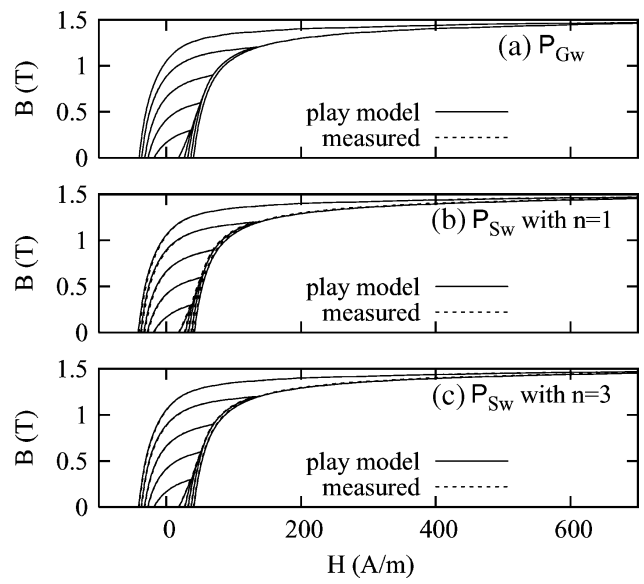

Fig. 4. Unidirectional property of vector models with $c=0.5$ : (a) $\boldsymbol{P}_{\mathrm{Gw}}$, (b) $\boldsymbol{P}_{\mathrm{Sw}}$ with $n=1$, and (c) $\boldsymbol{P}_{\mathrm{Sw}}$ with $n=3$.
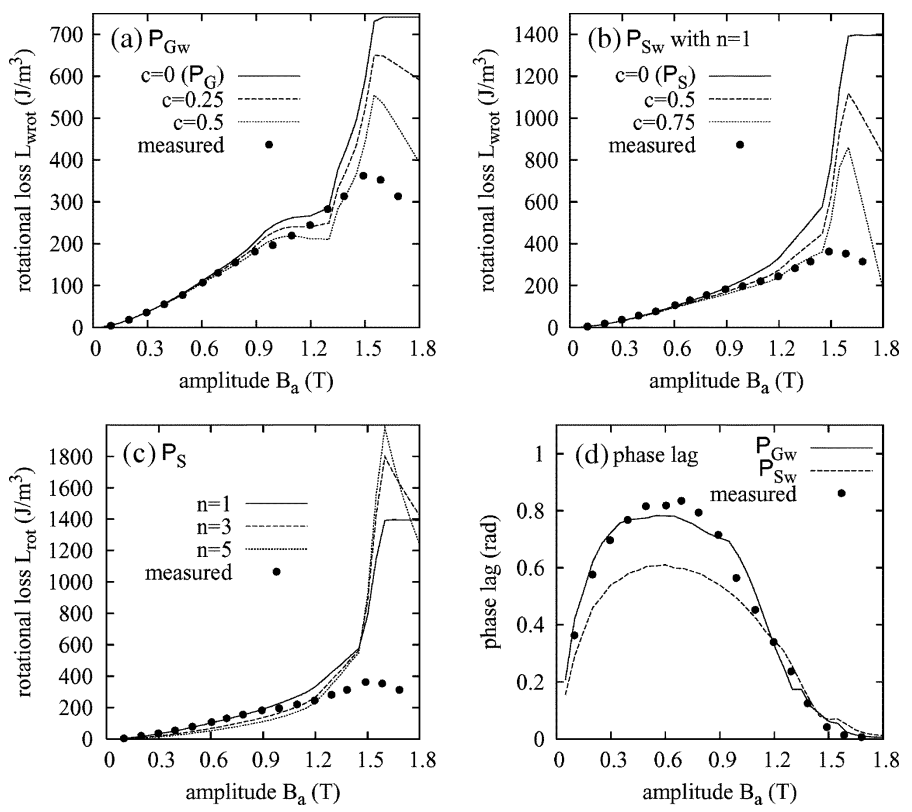

Fig. 5. Rotational properties of vector play models: (a) $L_{\text {wrot }}$ of $\boldsymbol{P}_{\mathrm{Gw}}$, (b) $L_{\text {wrot }}$ of $\boldsymbol{P}_{\mathrm{Sw}}$ with $n=1$, (c) $L_{\text {rot }}$ of $\boldsymbol{P}_{\mathrm{S}}$, and (d) phase lag.

gives a hysteresis loss that decreases with the increase in $B_{\mathrm{a}}$ for $B_{\mathrm{a}} \geq B_{\mathrm{S}}$. However, altering only $n$ is not effective for adjusting the simulated rotational hysteresis loss to that which has been measured. Fig. 5(a) and (b) shows that the weighting function significantly affects the rotational loss. However, the weighting function (19) is too simple to adjust $L_{\mathrm{wrot}}(B)$ to the measured rotational loss.

The vector model $\boldsymbol{P}_{\mathrm{Gw}}$ yields a smaller rotational hysteresis loss and larger phase lag than $\boldsymbol{P}_{\mathrm{Sw}}$. This means that $\boldsymbol{P}_{\mathrm{Gw}}$ has smaller amplitude $\boldsymbol{H}$ than $\boldsymbol{P}_{\mathrm{Sw}}$.

\section{Determination of Weighting Function}

A correlation between $w(B)$ and $L_{\mathrm{wrot}}$ can be used to adjust $L_{\mathrm{wrot}}$ to the measured rotational loss. For example, the weighting function is successively modified by

$$
w^{k+1}(B)=\frac{L_{\mathrm{rot}}^{\text {measured }}(B)}{L_{\mathrm{wrot}}^{k}(B)} w^{k}(B)
$$



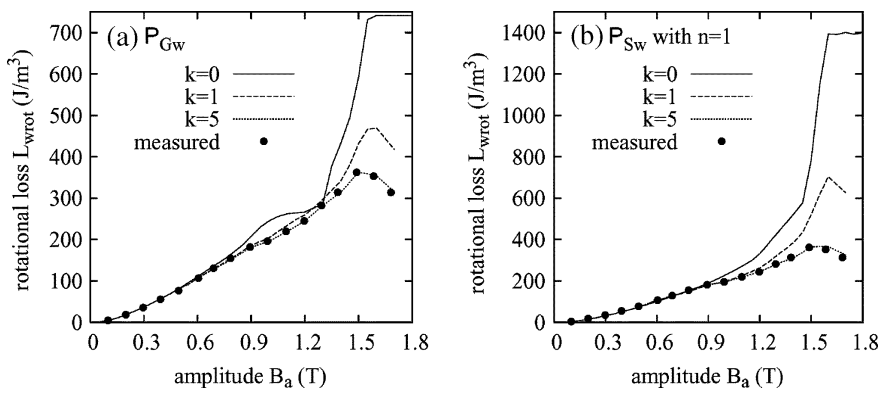

Fig. 6. Rotational loss given by $w(B)^{k}$ in (21): (a) $\boldsymbol{P}_{\mathrm{Gw}}$ and (b) $\boldsymbol{P}_{\mathrm{Sw}}$.

where $L_{\mathrm{wrot}}^{k}(B)$ is the computed rotational loss given by the weighting function for the $k$ th iteration and $w^{0}(B)=1$.

Fig. 5 shows that $L_{\mathrm{wrot}}(B)$ given by $w(B)=1(c=0)$ differs from the measured loss when $B_{\mathrm{a}}>0.8 \mathrm{~T}$. Accordingly, $w(B)$ is modified by (21) for $B>0.5 \mathrm{~T}$ while $w(B)=1$ for $B \leq 0.5$ T. Fig. 6 shows the rotational losses where $L_{\text {wrot }}^{5}(B)$ almost coincides with the measured loss. The phase lag of $\boldsymbol{B}$ to $\boldsymbol{H}$, however, was not much affected by this modification.

\section{CONCLUSION}

Two types of isotropic vector play models are discussed; the first is represented by a superposition of scalar play models, whereas the second is given by a geometrical vectorization of a play hysteron. The latter is more efficient than the former, which requires an integration along the azimuthal direction.

Both vector models are generalized with weighting functions to control their rotational hysteresis losses. The simulated rotational hysteresis loss can be adjusted to the measured loss by an iterative method to determine the weighting function.

\section{APPENDIX}

The scalar play model (1) is decomposed into an irreversible component $(\zeta>0)$ and a reversible component $(\zeta=0)$ [9] as

$$
P(B)=F(B)+\int_{+0}^{B_{S}} f\left(\zeta, p_{\zeta}(B)\right) \mathrm{d} \zeta
$$

where $F(B)=\lim _{\delta \rightarrow+0} \int_{0}^{\delta} f\left(\zeta, p_{\zeta}(B)\right) \mathrm{d} \zeta$ is the reversible component.

The vector play model (9) is identified from its unidirectional property. The unidirectional property of $\boldsymbol{P}_{\mathrm{Sw}} / w(|\boldsymbol{B}|)\left(=\boldsymbol{P}_{\mathrm{S}}\right)$ along $\theta=0$ is given by

$$
\frac{H}{w(B)}=P_{1}(B)=\int_{-\pi / 2}^{\pi / 2} P_{2}\left(B \cos ^{\frac{1}{n}} \varphi\right) \cos \varphi \mathrm{d} \varphi
$$

where $P_{1}$ is a scalar hysteretic property represented by a scalar model (1). The play model $P_{2}$ is identified from $P_{1}$ as follows.

The following function $Q_{k}$ is defined for identification:

$$
Q_{k}(\zeta, p)=F_{k}(p)+\int_{+0}^{\zeta} f_{k}(\xi, p) \mathrm{d} \xi
$$

In this definition, $F_{k}$ and $f_{k}(k=1,2)$ denote the reversible component and shape function of $P_{k}$, respectively. Equation (24) requires the following relation between $Q_{1}$ and $Q_{2}$ :

$$
Q_{1}(\zeta, p)=\int_{-\pi / 2}^{\pi / 2} Q_{2}\left(\zeta \cos ^{\frac{1}{n}} \varphi, p \cos ^{\frac{1}{n}} \varphi\right) \cos \varphi \mathrm{d} \varphi .
$$

This integral equation is solved for $Q_{2}$ by (26) and (27)

$$
\begin{aligned}
Q_{2}(\zeta, p) & =\frac{1}{\pi} \int_{0}^{\pi / 2} Q_{12}\left(\zeta \cos ^{\frac{1}{n}} \varphi, p \cos ^{\frac{1}{n}} \varphi\right) \mathrm{d} \varphi \\
Q_{12}(\zeta, p) & =Q_{1}(\zeta, p)+\frac{\zeta}{n} \frac{\partial Q_{1}(\zeta, p)}{\partial \zeta}+\frac{p}{n} \frac{\partial Q_{1}(\zeta, p)}{\partial p} .
\end{aligned}
$$

Functions $f_{2}$ and $F_{2}$ are obtained from $Q_{2}$ by (28)

$$
F_{2}(p)=Q_{2}(+0, p), f_{2}(\zeta, p)=\frac{\partial Q_{2}(\zeta, p)}{\partial \zeta}(\zeta>0) .
$$

\section{ACKNOWLEDGMENT}

The authors would like to express their gratitude to their graduate students H. Hirao and H. Kaneda, Kyoto University, Japan, for measurement of magnetic properties of silicon steel sheets. This work was supported in part by the Japan Society for the Promotion of Science, Grant-in-Aid for Scientific Research (C), 19560288.

\section{REFERENCES}

[1] M. A. Krasnosel'skii and A. V. Pokrovskii, Systems With Hysteresis. Berlin Heidelberg: Springer-Verlag, 1989.

[2] S. Bobbio, G. Miano, C. Serpico, and C. Visone, "Models of magnetic hysteresis based on play and stop hysterons," IEEE Trans. Magn., vol. 33, pp. 4417-4426, Nov. 1997.

[3] I. D. Mayergoyz, Mathematical Models of Hysteresis and Their Applications. New York: Elsevier, 2003.

[4] M. Brokate, "Some mathematical properties of the Preisach model for hysteresis," IEEE Trans. Magn., vol. 25, pp. 2922-2924, Jul. 1989.

[5] T. Matsuo and M. Shimasaki, "Representation theorems for stop and play models with input-dependent shape functions," IEEE Trans. Magn., vol. 41, pp. 1548-1551, May 2005.

[6] A. Bergqvist, "Magnetic vector hysteresis model with dry friction-like pinning," Physica B, vol. 233, pp. 342-347, 1997.

[7] C. Serpico, M. d'Aquino, C. Visone, and D. Davino, "A new class of Preisach-type isotropic vector model of hysteresis," Physica B, vol. 343, pp. 117-120, 2004.

[8] T. Matsuo, Y. Osaka, and M. Shimasaki, "Eddy-current analysis using vector hysteresis models with play and stop hysterons," IEEE Trans. Magn., vol. 36, pp. 1172-1177, Jul. 2000.

[9] T. Matsuo and M. Shimasaki, "An identification method for an isotropic vector hysteresis model constructed by the superposition of play models," in Preisach Memorial Book, A. Ivanyi, Ed. Budapest: Akadémiai Kiadó, 2005, pp. 39-50.

[10] N. Takahashi, S. Miyabara, and K. Fujiwara, "Problems in practical finite element analysis using Preisach hysteresis model," IEEE Trans. Magn., vol. 35, pp. 1243-1246, May 1999.

[11] T. Matsuo and M. Shimasaki, "An identification method of play model with input-dependent shape function," IEEE Trans. Magn., vol. 41, pp. 3112-3114, Oct. 2005.

[12] T. Matsuo, H. Hirao, and M. Shimasaki, "Preliminary study of 2-dimensional magnetic-property measurement of silicon steel sheet using stator of induction motor," Przeglad Elektrotechniczny, vol. 2007, pp. 67-69, Apr. 2007.

Manuscript received June 24, 2007. Corresponding author: T. Matsuo (e-mail: tmatsuo@kuee.kyoto-u.ac.jp). 